\title{
ORIGINAL
}

\section{Viscoelastic haemostatic assay augmented protocols for major trauma haemorrhage (ITACTIC): a randomized, controlled trial}

\author{
K. Baksaas-Aasen ${ }^{1}$, L. S. Gall ${ }^{2}$, J. Stensballe ${ }^{3}$, N. P. Juffermans ${ }^{4}$, N. Curry ${ }^{5}$, M. Maegele ${ }^{6}$, A. Brooks ${ }^{7}$, C. Rourke ${ }^{2}$, \\ S. Gillespie², J. Murphy ${ }^{8}$, R. Maroni ${ }^{8}$, P. Vulliamy², H. H. Henriksen³, K. Holst Pedersen³, K. M. Kolstadbraaten', \\ M. R. Wirtz ${ }^{4}$, D. J. B. Kleinveld ${ }^{4}$, N. Schäfer ${ }^{6}$, S. Chinna $^{7}$, R. A. Davenport ${ }^{2}$, P. A. Naess ${ }^{1}$, J. C. Goslings ${ }^{4}$, \\ S. Eaglestone' ${ }^{2}$ S. Stanworth ${ }^{5,9}$, P. I. Johansson ${ }^{3}$, C. Gaarder ${ }^{1}$ and K. Brohi ${ }^{*}$
}

(c) 2020 The Author(s)

\begin{abstract}
Purpose: Contemporary trauma resuscitation prioritizes control of bleeding and uses major haemorrhage protocols (MHPs) to prevent and treat coagulopathy. We aimed to determine whether augmenting MHPs with Viscoelastic Haemostatic Assays (VHA) would improve outcomes compared to Conventional Coagulation Tests (CCTs).

Methods: This was a multi-centre, randomized controlled trial comparing outcomes in trauma patients who received empiric MHPs, augmented by either VHA or CCT-guided interventions. Primary outcome was the proportion of subjects who, at $24 \mathrm{~h}$ after injury, were alive and free of massive transfusion (10 or more red cell transfusions). Secondary outcomes included 28-day mortality. Pre-specified subgroups included patients with severe traumatic brain injury (TBI).
\end{abstract}

Results: Of 396 patients in the intention to treat analysis, 201 were allocated to VHA and 195 to CCT-guided therapy. At $24 \mathrm{~h}$, there was no difference in the proportion of patients who were alive and free of massive transfusion (VHA: 67\%, CCT: 64\%, OR 1.15, 95\% Cl 0.76-1.73). 28-day mortality was not different overall (VHA: 25\%, CCT: 28\%, OR 0.84, 95\% Cl 0.54-1.31), nor were there differences in other secondary outcomes or serious adverse events. In pre-specified subgroups, there were no differences in primary outcomes. In the pre-specified subgroup of 74 patients with TBI, 64\% were alive and free of massive transfusion at $24 \mathrm{~h}$ compared to $46 \%$ in the CCT arm (OR 2.12, 95\% Cl 0.84-5.34).

Conclusion: There was no difference in overall outcomes between VHA- and CCT-augmented-major haemorrhage protocols.

Keywords: Trauma, Haemorrhage, Coagulopathy, Thrombelastography, Thromboelastometry

\footnotetext{
*Correspondence: k.brohi@qmul.ac.uk

${ }^{2}$ Centre for Trauma Sciences, Queen Mary University of London, Blizard Institute, 4 Newark Street, London E1 2AT, UK
}

Full author information is available at the end of the article

\section{勿 Springer}




\section{Introduction}

Major haemorrhage after trauma is estimated to be responsible for nearly half of the annual 4.6 million injury deaths worldwide [1]. Up to $50 \%$ of critically bleeding patients will die, either early from exsanguination, or later from multiple organ dysfunction or associated traumatic brain injury $[2,3]$.

Current approaches to trauma resuscitation focus on control of bleeding and management of traumainduced coagulopathy with the timely administration of haemostatic therapy [2,4]. In the initial phases of care, these therapies are often delivered empirically as part of a major haemorrhage protocol (MHP), delivering tranexamic acid, and blood components in proportions that approach the composition of whole blood [5, 6]. However, it is known that these strategies are rarely able to fully correct coagulopathy once established [7, 8], and all patients receive the same management approach, regardless of the severity or nature of their haemostatic deficits.

An alternative approach is a targeted and individualized approach to diagnosing and correcting coagulopathy. Conventional coagulation tests (CCTs) have been incorporated into algorithms but suffer from slow turnaround times and may lack the ability to guiding individual therapies [9]. We and others have investigated the diagnostic potential of point-of-care Viscoelastic Haemostatic Assays (VHAs) in trauma patients [9-15]. However, robust multi-centre and international randomized trial data on the effectiveness of VHA-guided haemostatic therapy is lacking in trauma [16-20]. Our earlier work in over 2000 trauma patients developed trauma-specific algorithms [21], and we now wished to determine whether the application of a targeted strategy in addition to standard-of-care would improve outcomes after a major trauma haemorrhage.

The "Implementing Treatment Algorithms for the Correction of Trauma-Induced Coagulopathy (ITACTIC)" trial was designed to determine the efficacy of augmenting empiric standard MHPs with VHA-guided interventions in improving outcomes from trauma haemorrhage, as compared to a standard MHP augmented by active CCT monitoring and guided interventions. We hypothesized that VHA-augmented MHPs would improve mortality and reduce the need for massive transfusion (ten or more units of red blood cell (RBC) transfusions) in the first $24 \mathrm{~h}$ after injury.

\section{Methods}

Trial design

The ITACTIC trial was a pragmatic, multi-centre, randomized controlled trial involving injured patients who

\section{Take-home message}

When standard of care is delivered with empiric balanced haemostatic therapy and intensive conventional coagulation testing, viscoelastic haemostatic assays did not improve clinical outcomes in the intention to treat cohort.

were suspected of having haemorrhage and who required at least one $\mathrm{RBC}$ transfusion. The trial compared outcomes in patients who received an empiric MHP supplemented by haemostatic therapy guided by either CCTs (CCT group) or by VHAs (VHA group). The trial was registered at ClinicalTrials.gov (NCT02593877, Nov 1st, 2015) and the protocol (Supplementary Materials) has previously been published [22].

Participating centres were members of the International Trauma Research Network (www.intrn.org) and were chosen on the basis of their ability to deliver the study design, expected enrolment rates, and the use of a standard empiric major haemorrhage protocol conforming to relevant national guidance and including balanced blood component therapy and empiric use of tranexamic acid. The trial was conducted at seven major trauma centres in Europe (Rigshospitalet (Copenhagen, Denmark), Amsterdam University Medical Centre (Amsterdam, The Netherlands), Oslo University Hospital (Oslo, Norway), Kliniken der Stadt Köln gGmbH (Cologne, Germany), The Royal London Hospital (London, UK), John Radcliffe Hospital (Oxford, UK) and Nottingham University Hospitals, Queens Medical Centre (Nottingham, UK)). The VHA device used at each study site (Thromboelastography-TEG 6S Haemostasis Analyzer, Haemonetics ${ }^{\circledR}$ Corporation, or Rotational Thromboelastometry-ROTEM ${ }^{\circledR}$ Sigma, TEM Innovations $\mathrm{GmbH}$ ) was determined by existing familiarity with a specific device appliance and to ensure a balanced use of the devices across the study.

The trial was designed by the authors, and the design was approved by the trial sponsor (Queen Mary University of London) and the national research ethics committees of participating institutions. Clinical trial oversight, monitoring and data management were conducted by the clinical studies unit of the Centre for Trauma Sciences, Queen Mary University of London. An independent Data Monitoring Committee and a Trial Steering Committee provided oversight of trial activities.

\section{Patient population}

Adult trauma patients were enrolled if they presented with clinical signs of bleeding activating the local MHP and if RBC transfusion had been initiated. Participants had to be randomized within $3 \mathrm{~h}$ of injury and maximum 
of $1 \mathrm{~h}$ after admission to the emergency department. There were no additional exclusion criteria.

Consent for initial enrolment in the trial was provided on behalf of incapacitated patients by a nominated consultee, usually an independent clinician in charge of patient care. Subsequent consent was given by a personal consultee (e.g. a family member) when identified and able to go through the informed consent process. Individual informed consent was subsequently obtained if the patient regained the physical and mental capacity to provide such consent. If the patient died or was otherwise unable to give consent, previous nominated professional or personal consultee consent remained in effect. Patients who subsequently withdrew their consent were removed from the trial.

\section{Randomization and blinding}

Enrolled patients were randomized in a 1:1 ratio to the VHA or the CCT groups, with block randomization by the centre. Randomization codes were generated and secured by an independent statistician. Block size was unknown to on-site study teams. Group allocation was by study personnel opening the numbered opaque sealed envelope in sequence taken from a stack held by each study site. Once an envelope had been opened, the subject was considered enrolled. The trial was un-blinded to the treating clinical teams, while research personnel collecting subsequent safety and outcome data were blinded to group allocation.

\section{Procedures}

All patients received their local hospital's standard MHP, based on the empiric delivery of tranexamic acid; blood components delivered in a 1:1:1 ratio of RBCs, plasma and platelet transfusions; and limited infusion of crystalloid fluids. In both groups, blood was drawn for coagulation analysis at baseline and after every four units of RBCs transfused, until haemostasis. In the CCT group, these were the conventional coagulation tests performed in the laboratory, while in the VHA assays, analyses were performed at the point of care. Haemostatic therapy was delivered based on results of these according to the TACTIC algorithms [21], which define triggers for additional administration of platelet, fibrinogen, plasma and antifibrinolytic therapies.

\section{Definitions}

The time of haemostasis was defined as $1 \mathrm{~h}$ after the last $\mathrm{RBC}$ transfusion was given and the treating clinicians stated haemostasis had been achieved. Massive transfusion was defined as the administration of ten or more units of RBCs in the first $24 \mathrm{~h}$ after injury. Severe traumatic brain injury (TBI) was defined as an Abbreviated
Injury Scale score in the brain of four, five, or six [23]. We used an anatomical head injury score rather than an assessment of admission level of consciousness as the latter is affected by the depth of shock in this group of patients. We used prothrombin time ratio (PTr) as a test of coagulation available in both arms of the trial [24] and defined PTr $>1.2$ as abnormal. For reporting blood component therapy across study centres, one pool of cryoprecipitate was considered equivalent to two grams of fibrinogen concentrates (or fibrinogen equivalent dose in grams); and platelet concentrates are reported as administered in pooled components (one pool=four individual platelet units).

\section{Outcomes}

The primary outcome of this study was the proportion of subjects who, at $24 \mathrm{~h}$ after injury, were alive and free of massive transfusion. Secondary endpoints included: all-cause mortality at $6 \mathrm{~h}, 24 \mathrm{~h}, 28$ days, and 90 days post-admission; total blood components; 28 -day ventilator-free, and intensive care unit (ICU)-free days; total hospital length-of-stay; and the proportion of patients with symptomatic thromboembolic events, with multiple organ dysfunction, and with serious adverse events.

\section{Statistical analysis}

The planned sample size for this study was 392, 196 in each study arm. Prior data from the study centres estimated that approximately $28 \%$ of patients die or receive a massive transfusion by $24 \mathrm{~h}$. Based on an estimated reduction in this proportion to $15 \%$ in the VHA group, and with a significance level of 0.05 using a two-sided test, 170 patients per group were required to achieve $80 \%$ power, increasing to 196 per group to account for a predicted drop-out rate of $15 \%$.

All primary and secondary outcomes were analysed as intention-to-treat and per-protocol. The per-protocol group was defined excluding patients who did not have at least one VHA or CCT test performed, who did not meet the inclusion criteria, who received the wrong test, who died within $60 \mathrm{~min}$ after baseline blood sampling or who achieved haemostasis within $60 \mathrm{~min}$ of baseline sampling.

The primary endpoint was assessed by logistic regression to produce an odds ratio with $95 \%$ confidence interval. Mortality was compared between the two arms as a binary outcome by logistic regression and using KaplanMeier methods and the log-rank test. Time to haemostasis, 28-day ventilator-free and ICU-free days, total hospital length-of-stay, EQ-5D quality-of-life scores, total number of blood products transfused, numbers receiving study intervention (post-hoc analysis) and time to intervention (post-hoc analysis) were compared using 
the Wilcoxon-Mann-Whitney or Chi-square tests. Missing data for the primary outcome were not expected. For secondary outcomes, data were assumed to be missing at random, therefore participants with missing data for a measure were excluded from any statistical comparisons regarding that measure. Denominators for analyses are presented throughout.

Pre-specified subgroups of interest were patients with and without severe TBI (defined as having an injury to the head of Abbreviated Injury Scale score of 4 or more); patients with a prolonged $\mathrm{PTr}$ at baseline; patients receiving a massive transfusion; and patients on prior oral anticoagulant therapies. Stratification within subgroups was not possible as laboratory and imaging results were not available at the time of randomization and enrolment. The pre-defined subgroups were analysed by intention-to-treat for the primary outcome (except for the patients receiving a massive transfusion as not applicable) and some secondary outcomes (posthoc analyses), such as 28-day mortality, total number of blood products, numbers receiving study intervention and time to intervention. As a comparison with the opposite subgroup, patients without a prolonged PTr were also analysed (post-hoc analysis). Interaction p-values were calculated (post-hoc analysis) to inspect the effect of each subgroup on the outcome of interest. The primary endpoint and 28-day mortality analyses for the severe TBI subgroup were also performed adding covariates to the logistic regressions to adjust for any risk differences between study arms at baseline (post-hoc analysis). Statistical analyses were performed using Stata version 13. All applied tests were two-sided and $p$-values of less than 0.05 were considered statistically significant. A pre-defined interim analysis was performed after the enrolment of 100 patients and the Data Monitoring Committee reviewed all data on outcomes for every 50 patients enrolled. The statistical analyses plan is presented in the Supplementary Materials.

\section{Results}

From 1st of June 2016 to 30th of July 2018, 480 trauma patients were eligible for enrolment, of which 411 were randomized. 15 patients subsequently withdrew consent, leaving 396 subjects for the intention-to-treat analysis (Fig. 1): 201 patients were allocated to the VHA group and 195 to the CCT group. Baseline characteristics were well matched between treatment groups (Table 1). Two-thirds of the intention-to-treat cohort had sustained blunt trauma alone, and the overall median Injury Severity Score (ISS) was 26 (interquartile range (IQR) 17-36, with a score over 15 indicating severe trauma). The median time from injury to admission was $69 \mathrm{~min}$ (IQR 45-96 min). At enrolment, participants had already received a median of two units of RBCs (IQR 1-4) and 9 had already received massive transfusion (ten or more units of RBCs). Of the 377 patients who received tranexamic acid, 268 (71\%) received the $1 \mathrm{~g}$ bolus prior to enrolment.

After enrolment and before haemostasis, 120/178 (67\%) patients in the VHA group received a study intervention, compared to $62 / 170(36 \%)$ in the CCT group (Supplementary Table S1). The study interventions were given a median of 21 min earlier in the VHA group (VHA $61 \mathrm{~min}$, IQR 48-85; CCT $80 \mathrm{~min}$, IQR 60-106, Supplementary Table S1), with 89/139 (64\%) of patients in the VHA group receiving the first study intervention within $3 \mathrm{~h}$ of injury compared to $45 \%$ (39/86) in the CCT group. Between baseline and haemostasis, patients received a median of three units of RBCs and four units of plasma in both arms of the trial (Supplementary Table S1). Within this period, patients in the VHA arm received more fibrinogen supplementation (median fibrinogen equivalent dose-VHA: $4 \mathrm{~g}$, IQR 0-4; CCT: 0 g, IQR 0-4 Supplementary Table S1).

At $24 \mathrm{~h}$ after injury, there was no difference between the two groups in the proportion of patients who were alive and free of massive transfusion (VHA 67\%, CCT $64 \%$, OR 1.15, 95\% CI 0.76-1.73, Fig. 2). In the VHA arm, 29/201 (14\%) of patients had died and 53/201 (26\%) had received a massive transfusion at $24 \mathrm{~h}$, while in the CCT arm, 33/195 (17\%) of patients had died and $55 / 195(28 \%)$ had received a massive transfusion (Table 2). At 28 days, $50 / 201$ (25\%) of patients in the VHA arm and 55/194 (28\%) patients in the CCT arm had died (OR 0.84, 95\% CI 0.54-1.31, Table 2 and Supplementary Figure S1). This pattern persisted to 90 days (Fig. 3).

There were no statistically significant differences in other secondary outcomes between the two study groups, including the rate of multiple organ dysfunction, the incidence of symptomatic thromboembolic events, and a number of ventilator-free days or ICU-free days at day 28 (Table 2). Hospital length-of-stay was also similar in both groups and so were quality-of-life (EQ-5D) scores at both discharge/28 days and 90 days (Table 2). Safety and cause-of-death profiles were similar across the two groups (Table 3).

For the per-protocol analysis, 83 patients were excluded from the ITT cohort: five for not meeting the entry criteria, eight for receiving the wrong treatment, and 54 for reaching haemostasis and 16 for dying within the first hour (Fig. 1). This left 313 patients in the perprotocol cohort (150 VHA, 163 CCT, Fig. 1). There was no statistically significant difference between study arms for the primary outcome or for 28-day mortality in the per-protocol group (Fig. 2 and Supplementary Fig. S1). 


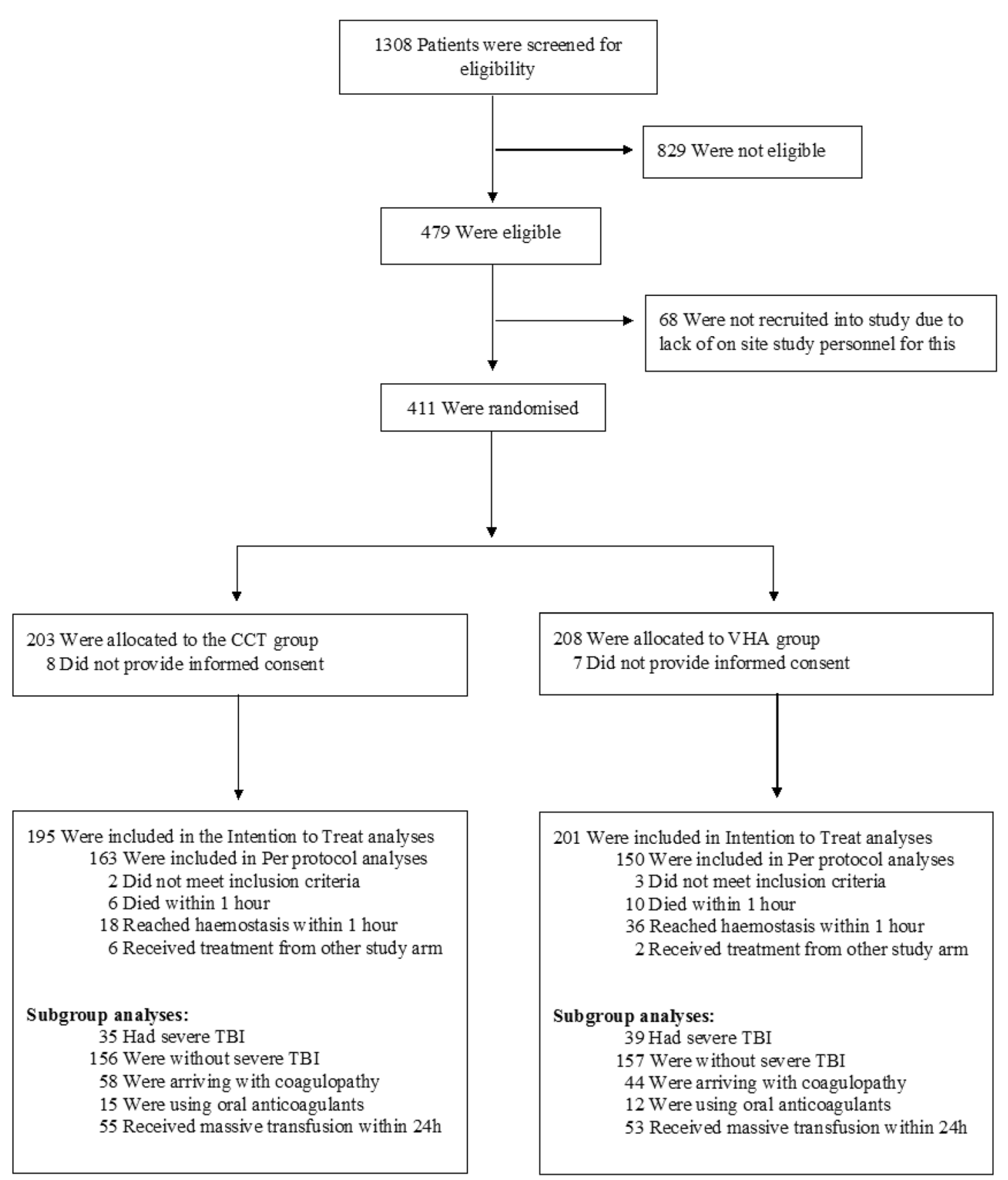

Fig. 1 CONSORT diagram. CCT conventional coagulation test, VHA Viscoelastic Haemostatic Assay, TBI traumatic brain injury

Across the pre-specified subgroups, there was no statistically significant difference in the primary outcome (Fig. 2). In the subgroup of 74 patients who presented with severe TBI, 25/49 (64\%) patients in the VHA arm were alive and free of massive transfusion at $24 \mathrm{~h}$, compared to $16 / 35(46 \%)$ in the CCT arm (OR 2.12 95\% CI 0.84-5.34). After risk adjustment for admission characteristics, this odds ratio was unchanged at 2.10 (95\% CI 0.51-8.61, Supplemental Table S3). Additional analyses on secondary outcomes of the TBI and other subgroups are presented in the Supplementary Materials (Fig. S1, Tables S1-S3). 
Table 1 Demographics, injuries and admission characteristics and initial therapies (intention-to-treat population)

\begin{tabular}{|c|c|c|}
\hline & $\mathrm{CCT}(n=195)$ & VHA $(n=201)$ \\
\hline Median age (IQR) & $43(28-59), n=194$ & $40(26-54), n=197$ \\
\hline Male sex - no. (\%) & 159/194 (82\%) & 145/198 (73\%) \\
\hline Prior oral anticoagulation - no. (\%) & 15/192 (8\%) & $12 / 198(6 \%)$ \\
\hline \multicolumn{3}{|l|}{ Injuries \& Admission Physiology } \\
\hline Injury caused by blunt trauma alone^ - no. (\%) & $130 / 194(67 \%)$ & $133 / 198(66 \%)$ \\
\hline Median Injury Severity Score (IQR) & $26(16-35), n=191$ & $26(17-37), n=196$ \\
\hline Severe TBI (AIS Head > 3) - no. (\%) & $35 / 191(18 \%)$ & $39 / 201(20 \%)$ \\
\hline Median time from injury to admission (IQR) - min & $67(42-98), n=191$ & $70(48-95), n=195$ \\
\hline Median heart rate (IQR) - beats/min & $105(82-123), n=181$ & $103(87-127), n=190$ \\
\hline Median systolic blood pressure (IQR) - mmHg & $90(74-110), n=170$ & $95(73-120), n=178$ \\
\hline Median Glasgow Coma Scale score (IQR) & $13(3-15), n=191$ & $12(3-15), n=194$ \\
\hline Patients with PTr > 1.2 - no. (\%) & $58 / 181(32 \%)$ & $44 / 175(25 \%)$ \\
\hline Median base deficit (IQR) - mEq/L & $7.2(4.2-13), n=188$ & $8.2(4.4-12.8), n=190$ \\
\hline Median lactate (IQR) - mEq/L & $4.4(2.8-8.2), n=185$ & $4.5(2.9-8), n=191$ \\
\hline Median fibrinogen level (IQR) - g/L & $2.0(1.4-2.4), n=n=177$ & $1.9(1.5-2.4), n=166$ \\
\hline \multicolumn{3}{|l|}{ Pre-baseline therapy } \\
\hline Received tranexamic acid bolus—no. (\%) & 190/193 (98\%) & $187 / 200(94 \%)$ \\
\hline Median units of RBCs (IQR) & $2(1-4), n=179$ & $2(1-4), n=n=187$ \\
\hline Median units of FFP/Octaplasma (IQR) & $0(0-2), n=179$ & $0(0-2), n=187$ \\
\hline Median equivalent dose of fibrinogen (IQR) - $g$ & $0(0-0), n=184$ & $0(0-0), n=185$ \\
\hline Median units of Platelets (IQR) & $0(0-0), n=179$ & $0(0-0), n=n=187$ \\
\hline
\end{tabular}

$\overline{C C T}$ conventional coagulation test, VHA viscoelastic haemostatic assay, IQR interquartile range, $T B /$ traumatic brain injury, AIS abbreviated injury scale, $P T r$ prothrombin time ratio, $R B C$ red blood cell, FFP fresh frozen plasma

$\wedge$ The other categorisations used for a type of trauma were: penetrating; or blunt and penetrating combined

\section{Discussion}

Our international randomized trial has evaluated the role of VHAs to individualize care and augment empiric haemostatic resuscitation in the challenging and timecritical environment of trauma care. Empiric haemostatic therapy was delivered in both arms of the trial, with balanced blood component transfusions, use of tranexamic acid and restricted crystalloid infusions. Intensive coagulation monitoring was also performed in both arms after every four units of red cell transfusions, with additional haemostatic therapies delivered based on these results. We saw no difference overall in primary or secondary outcomes between CCT and VHA-guided haemostatic therapy.

While this study aimed to explore the effect of VHAdirected assessment and treatment of coagulopathy in trauma patients, the overall prevalence of coagulopathy was lower than we expected. Nearly three quarters of the patients were not coagulopathic (by PTr) at baseline and very few of these patients subsequently developed a prolonged PTr before haemostasis. These lower rates are likely due to the effect of optimisation of balanced haemostatic therapy with early prehospital and in-hospital use of transfusions prior to randomisation and during haemorrhage. This alone was not sufficient to prevent trauma-induced coagulopathy, as coagulation deficits identified by CCTs resulted in treatment in $36 \%$ of patients in this arm of the trial (Supplementary Table S1). A previous single-centre trauma study reported a larger benefit with VHA-directed therapy, but subjects in the control arm received less empiric haemostatic transfusion therapy and the role of CCT monitoring during bleeding was not reported [25]. For those patients who never developed a coagulopathy, it is unsurprising that coagulation monitoring did not alter the clinical outcome. The relatively high numbers of these patients in our study reduced our ability to detect a difference in clinical outcomes.

Despite the delivery of optimized and individualised standard of care in both cohorts, $67 \%$ of patients in the VHA-augmented cohort received study interventions, 1.8 times more than in the CCT group. This indicates the widespread occurrence of coagulation deficits which were not detected by the CCTs. We are unable to ascertain in this analysis if these deficits were corrected by the directed therapies or whether the doses prescribed within the algorithms were too low. 


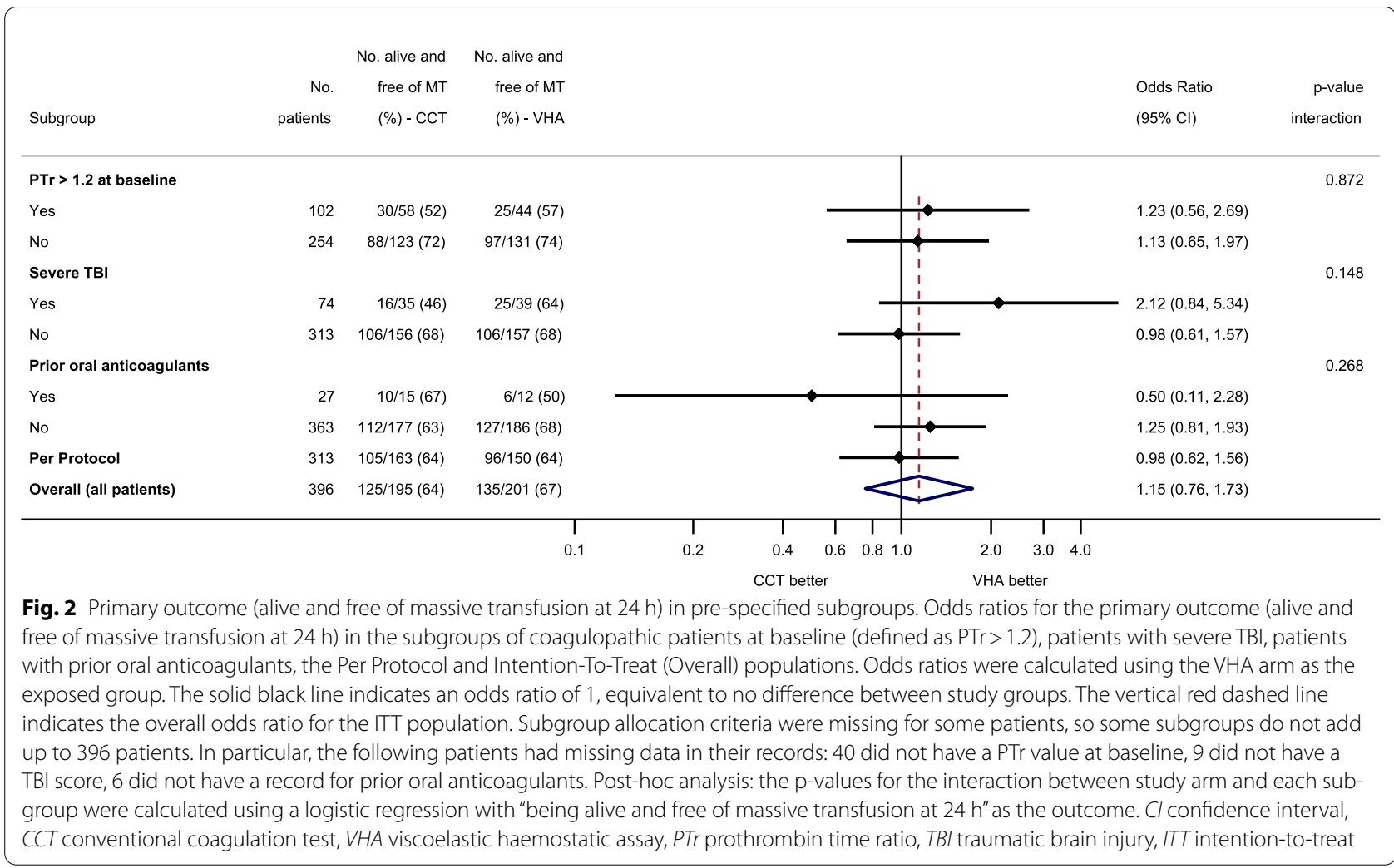

The reduction in 28-day mortality in the VHA group observed in patients who also had severe TBI was unexpected and may have been a chance finding. However, if borne out in future work, it potentially represents an important opportunity for improving outcomes in trauma care [26]. TBI is responsible for at least $25 \%$ of deaths in critically bleeding patients, and $50 \%$ of all trauma deaths [2]. Most previous clinical trials in trauma haemorrhage have excluded severe brain injuries as their outcomes were felt to be immutable by changes in haemostatic management. There is a small but growing body of evidence that directed coagulopathy management may reduce brain injury mortality, such as the two recent trials of tranexamic acid for TBI [27, 28]. Correction of coagulopathy may act to reduce intracerebral bleeding; decrease cerebral ischaemia due to reduced depth and duration of haemorrhagic shock; and/or reduce cerebral inflammation via crosstalk mechanisms, all of which constitute important areas for future brain injury research. The ITACTIC results are consistent with these hypotheses, and suggests this is an area for research in a field which has mostly focused on cerebral oxygenation and perfusion, and where there has been little change in practice or improvement in outcomes for some time [29-32].

This study was limited in a number of ways, including the lower observed difference in effect size in the primary endpoint between the two cohorts than originally predicted (observed: 3\%, predicted: 13\%). Empiric delivery of balanced transfusion and haemostatic therapies prior to randomisation and during haemorrhage likely contributed to this. Another caveat is that we did not adjust our secondary endpoint and post-hoc analyses for multiple comparisons due to the preponderance of non-significant results. As viscoelastic testing was only performed in the VHA group, we could not identify all coagulopathic patients and could not explore the effect of targeted therapies in coagulation deficits that were only detectable by VHA. Interpretation of the results from our subgroups is also limited by lack of group allocation stratification, which was impossible with this study design, as neither coagulation status nor the presence of severe brain injury could be known prior to randomisation. The study was conducted at large major trauma centres, with experience of the use of VHA devices in a research setting, although most did not have VHA-guided care in routine clinical use at the start of the trial. The study was challenging to deliver on many levels and the experience in other centres may be different.

When standard care is delivered to bleeding trauma patients, with empiric balanced transfusion therapy and intensive CCT monitoring, VHAs identify more coagulation deficits and deliver additional haemostatic 
Table 2 Secondary outcomes for the intention-to-treat population

\begin{tabular}{|c|c|c|c|c|}
\hline & $\mathrm{CCT}(n=195)$ & VHA $(n=201)$ & Odds ratio $(95 \% \mathrm{Cl})$ & $p$ value \\
\hline Mortality at 6 h-no. (\%) & $22 / 195(11 \%)$ & $22 / 201(11 \%)$ & $0.97(0.52-1.80)$ & 0.915 \\
\hline Mortality at 24 h-no. (\%) & $33 / 195(17 \%)$ & 29/201 (14\%) & $0.83(0.48-1.42)$ & 0.495 \\
\hline Mortality at 28 days_-no. (\%) & $55 / 194(28 \%)$ & $50 / 201(25 \%)$ & $0.84(0.54-1.31)$ & 0.435 \\
\hline Mortality at 90 days-no. (\%) & $56 / 177(31 \%)$ & $53 / 179(29 \%)$ & $0.91(0.58-1.42)$ & 0.678 \\
\hline Death from exsanguination—-no. (\%) & $17 / 56(30 \%)$ & $13 / 51(25 \%)$ & $0.78(0.34-1.82)$ & 0.576 \\
\hline Died before haemostasis—no. (\%) & $24 / 54(44 \%)$ & $19 / 50(38 \%)$ & $0.77(0.35-1.67)$ & 0.505 \\
\hline Median time to haemostasis^ (IQR)—mins & $122(80-185), n=170$ & $125(77-185), n=176$ & & 0.929 \\
\hline PTr $>1.2$ at haemostasis^—no. (\%) & $17 / 151(11 \%)$ & $21 / 142(15 \%)$ & $1.37(0.70-2.69)$ & 0.369 \\
\hline Massive transfusion at $24 \mathrm{~h} —$ no. (\%) & $55 / 195(28 \%)$ & $53 / 201(26 \%)$ & $0.91(0.59-1.42)$ & 0.682 \\
\hline Patients with symptomatic TE^^—no. (\%) & 27/195 (14\%) & $17 / 201(9 \%)$ & $0.57(0.31-1.08)$ & 0.088 \\
\hline Patients with MODS^^^—no. (\%) & 134/159 (84\%) & $141 / 164(86 \%)$ & $1.14(0.62-2.10)$ & 0.668 \\
\hline Median 28-day ventilator-free days (IQR) & $20(0-26), n=192$ & $17(0-25), n=198$ & & 0.422 \\
\hline Median 28-day ICU-free days (IQR) & $15(0-23), n=192$ & $13(0-23), n=198$ & & 0.691 \\
\hline Median hospital LOS in survivors (IQR) & $24(10-42), n=138$ & $29(13-49), n=147$ & & 0.147 \\
\hline 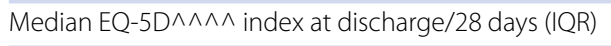 & $49(25-60), n=86$ & $40(28-60), n=n=92$ & & 0.672 \\
\hline 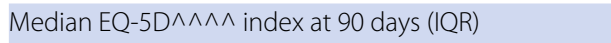 & $60(40-70), n=75$ & $53(40-70), n=72$ & & 0.718 \\
\hline
\end{tabular}

Proportions were calculated excluding any missing records

CCT conventional coagulation test, VHA viscoelastic haemostatic assay, Cl confidence interval, PTr prothrombin time ratio, TE thromboembolic events, MODS multiple organ dysfunction syndrome, ICU intensive care unit, LOS length of stay

$\wedge$ Patients with haemostasis (CCT: $n=n=170$, VHA: $n=178$ )

$\wedge \wedge$ Myocardial infarction and Embolic strokes are included in thromboembolic events

$\wedge \wedge \wedge$ Multiple Organ Dysfunction Syndrome (MODS) defined as having a Sequential Organ Failure Assessment score of 6 or more on a day $\wedge \wedge \wedge \wedge$ EQ-5D is a questionnaire by EuroQol used as a measure of overall health status

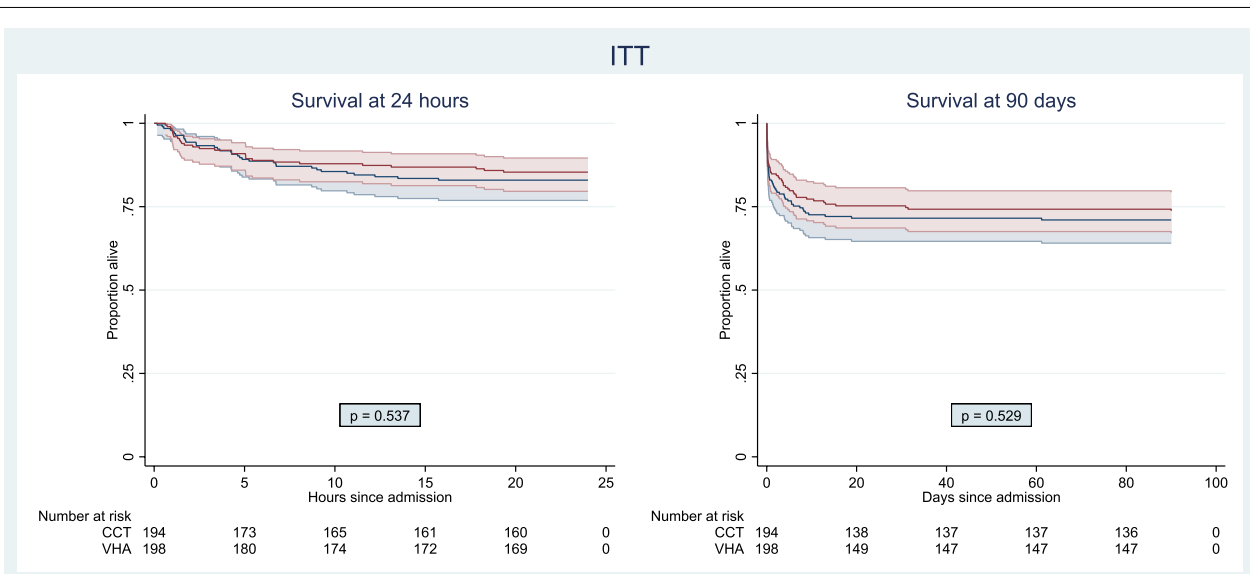

Fig. 3 Survival curves at $24 \mathrm{~h}$ and 90 days. Survival curves with $95 \%$ confidence intervals at $24 \mathrm{~h}$ and 90 days for the Intention-To-Treat (ITT) population. Blue: CCT-guided and Red:VHA-guided. The $p$ values shown are the result of the log-rank test. 4 patients in the ITT population had missing date/time of events and were therefore not included in the survival curve. CCT conventional coagulation test, VHA viscoelastic haemostatic assay 
Table 3 Distribution of serious adverse events (SAEs) and causes of death

\begin{tabular}{|c|c|c|c|c|}
\hline \multirow[t]{2}{*}{ SAE description^^ } & \multicolumn{2}{|l|}{$\begin{array}{l}\text { CCT } \\
(n=195)\end{array}$} & \multicolumn{2}{|l|}{$\begin{array}{l}\text { VHA } \\
(n=201)\end{array}$} \\
\hline & Events & Patients & Events & Patients \\
\hline Infection & $34(34 \%)$ & $30(32 \%)$ & $35(31 \%)$ & $29(29 \%)$ \\
\hline Thromboembolic^^ & $24(24 \%)$ & $22(24 \%)$ & $16(14 \%)$ & $15(15 \%)$ \\
\hline Ischemic & $0(0 \%)$ & $0(0 \%)$ & $6(5 \%)$ & $6(6 \%)$ \\
\hline Organ failure & $5(5 \%)$ & $5(5 \%)$ & $9(8 \%)$ & $9(9 \%)$ \\
\hline Acute kidney injury & $6(6 \%)$ & $6(6 \%)$ & $6(5 \%)$ & $6(6 \%)$ \\
\hline Acute lung injury & $6(6 \%)$ & $5(5 \%)$ & $12(11 \%)$ & $8(8 \%)$ \\
\hline New onset major bleeding & $9(9 \%)$ & $9(10 \%)$ & $6(5 \%)$ & $6(6 \%)$ \\
\hline Cardiac & $6(6 \%)$ & $6(6 \%)$ & $10(9 \%)$ & $10(10 \%)$ \\
\hline Neurological & $0(0 \%)$ & $0(0 \%)$ & $4(4 \%)$ & $4(4 \%)$ \\
\hline Other & $11(11 \%)$ & $10(11 \%)$ & $8(7 \%)$ & $8(8 \%)$ \\
\hline Total & $101(100 \%)$ & $93(100 \%)$ & $112(100 \%)$ & $101(100 \%)$ \\
\hline Cause of Death & & Patients & & Patients \\
\hline Uncontrolled Bleeding & & $17(30 \%)$ & & $13(25 \%)$ \\
\hline Head Injury & & $19(34 \%)$ & & $16(30 \%)$ \\
\hline Multiple Organ Dysfunction & & $6(11 \%)$ & & $10(19 \%)$ \\
\hline Thromboembolism & & $0(0 \%)$ & & $2(4 \%)$ \\
\hline Multiple Injuries & & $4(7 \%)$ & & $1(2 \%)$ \\
\hline Other & & $10(18 \%)$ & & $9(17 \%)$ \\
\hline Unknown & & $0(0 \%)$ & & $2(4 \%)$ \\
\hline Total & & 56 (100\%) & & $53(100 \%)$ \\
\hline
\end{tabular}

Serious Adverse Events (SAEs) were defined as any adverse event, adverse transfusion reaction or unexpected adverse transfusion reaction that resulted in death, was life-threatening, required hospitalization or prolongation of existing hospitalization, resulted in persistent or significant disability or incapacity, resulted in a congenital anomaly/birth defect or other medically significant event

$\wedge$ In the event field, SAEs experienced multiple times by the same patient are included in the count. In the patients field, patients experiencing multiple SAEs of the same type are included only once per type of SAE

$\wedge \wedge$ Myocardial infarction and embolic stroke are included in thromboembolic SAEs

interventions. However, all patients do not benefit from this approach and further research is required to identify injury types and physiologies that may benefit from this approach. Additional analyses should also explore the coagulation deficits identified by VHA alone, and the response of the coagulation system to the algorithm-prescribed haemostatic agents.

\section{Electronic supplementary material}

The online version of this article (https://doi.org/10.1007/s00134-020-06266-1) contains supplementary material, which is available to authorized users.

\section{Author details}

${ }^{1}$ Oslo University Hospital \& University of Oslo, Oslo, Norway. ${ }^{2}$ Centre for Trauma Sciences, Queen Mary University of London, Blizard Institute, 4 Newark Street, London E1 2AT, UK. ${ }^{3}$ Copenhagen University Hospital, Rigshospitalet, Copenhagen, Denmark. ${ }^{4}$ Amsterdam University Medical Centres, Amsterdam, The Netherlands. ${ }^{5}$ Oxford University Hospital NHS Trust, Oxford, UK. ${ }^{6}$ Cologne-Merheim Medical Centre, University of Witten/Herdecke, Cologne, Germany. ${ }^{7}$ Nottingham University Hospitals NHS Trust, Nottingham, UK. ${ }^{8}$ Queen Mary University of London, London, UK. ${ }^{9}$ NHS Blood and Transplant, Bristol, UK.

\section{Acknowledgements}

We would like to thank all the patients and their families who participated in this project. We are grateful for the contributions to the study of the Trial Steering Committee: Beverley Hunt (Chair), Heidi Doughty, Louis Riddez, Leo Geeraedts, Alison Rogers; and the Data Monitoring Committee: Ravi Gill (Chair), Jan Jansen and Phil Edwards.We would also like to acknowledge Joao Dias and Jan Hartmann (Haemonetics ${ }^{\circledR}$ Corporation) and Klaus Görlinger (TEM International $\mathrm{GmbH}$ ) for their personal support for the entire TACTIC program. We also acknowledge Amar Ahmed for early statistical support to the trial.This study is funded by the European Commission under the FP-7 HEALTH-Contract No. F3-2013-602771, entitled "Targeted Action for Curing Trauma Induced Coagulopathy" (TACTIC).Website European Commission FP-7 HEALTH-Contract No. F3-2013-602771: http://cordis.europa.eu/project/ rcn/110071_en.html.

\section{Author contributions}

Study design, development and study set up were performed by LSG, KB-A, JS, NPJ, NC, MM, CR, PV, RAD, PAN, JCG, SE, SS, PIJ, CG, KB. Site set up, subject enrolment, data collection and research governance were performed by KB-A, LSG, JS, NPJ, NC, MM, CR, PV, RAD, PAN, JCG, SE, SS, PIJ, CG and KB. RM and JM performed the statistical analysis. Initial draft of the manuscript was by KB-A, $\mathrm{RM}$ and $\mathrm{KB}$. All authors reviewed and commented on this and subsequent versions of the manuscript. AB, SC, SG, HHH, KHP, KMK, MRW, DJBK and NS contributed in data collection and commented on the manuscript. All authors vouch for the accuracy and completeness of the data and analyses, and for the fidelity of the trial to the protocol. No one who is not an author participated in the writing or editing of the manuscript. 


\section{Funding}

The study was part of the "Targeted Action for Curing Trauma-Induced Coagulopathy" (TACTIC) program, funded by the European Commission under the FP7 framework (Grant No. F3-2013-602771). Both TEM ${ }^{\circledR}$ International GmbH and Haemonetics ${ }^{\circledR}$ Corporation were collaborating organizations in the TACTIC program. For this ITACTIC study, they provided the ROTEM Sigma and TEG $6 \mathrm{~S}$ analyzers and all reagents to the relevant participating institutions. Neither these organizations nor any of their representatives had any role in the study design, data collection and analysis, decision to publish, or preparation of the manuscript.

\section{Compliance with ethical standards}

\section{Conflicts of interest}

K.Baksaas-Aasen, L.S.Gall, J.Stensballe, N.P.Juffermans, N.Curry, C.Rourke, S.Gillespie, J.Murphy, R.Maroni, P.Vulliamy, H.H.Henriksen, K.Holst Pedersen, K.M. Kolstadbraaten, M.R.Wirtz, D.J.B. Kleinveld, N.Schäfer, S.Chinna, P.A.Naess, J.C.Goslings, S.Eaglestone and S.Stanworth declare no conflicts of interest. M.Maegele has received honoraria for lectures and speakers' bureaus, congress travel support as well as financial support for research projects from Astra Zeneca, Bayer, CSL Behring, IL-Werfen/TEM International, LFB Biomedicaments and Portola Inc. A. Brooks has received a research grant from Haemonetics Corp. in the form of cartridges and reagent support. He has also served on advisory panel for Haemonetics Corp. and TEM International and received honoraria for education lectures for Johnson and Johnson. P.I. Johansson's has received unrestricted research grants from Haemonetics Corp. and Octapharma AG. C.Gaarder has received honoraria for lectures from Octapharma and research grant support from Haemonetics and TEM International in the form of device and reagent support. She has also previously served on the advisory board for Nycomed. K.Brohi and R.Davenport have received research grant support from TEM International in the form of device and reagent support. K.Brohi has previously served on external advisory panels for Haemonetics Corp, TEM International, CSL Behring, Bayer and Astra Zeneca.

\section{Ethical approval}

The study was designed by the authors, and the design was approved by the trial sponsor (Queen Mary University of London) and the national research ethics committees of participating institutions. The study was in accordance with the 1964 Helsinki Declaration.

\section{Consent to participate}

Initial enrolment in the trial was provided on behalf of incapacitated patients by a personal or nominated consultee until the patient regained the physical and mental capacity to provide such consent.

\section{Availability of data and material}

De-identified individual participant data underlying primary and secondary endpoints will be available on request from researchers, after approval of proposal. Study protocol and statistical analysis plan are presented in the Supplementary Materials, and informed consent form will be shared, if requested. The data will be available by contacting the corresponding author after publication.

\section{Open Access}

This article is licensed under a Creative Commons Attribution-NonCommercial 4.0 International License, which permits any non-commercial use, sharing, adaptation, distribution and reproduction in any medium or format, as long as you give appropriate credit to the original author(s) and the source, provide a link to the Creative Commons licence, and indicate if changes were made. The images or other third party material in this article are included in the article's Creative Commons licence, unless indicated otherwise in a credit line to the material. If material is not included in the article's Creative Commons licence and your intended use is not permitted by statutory regulation or exceeds the permitted use, you will need to obtain permission directly from the copyright holder. To view a copy of this licence, visit http://creativecommons.org/licen ses/by-nc/4.0/.

\section{Publisher's Note}

Springer Nature remains neutral with regard to jurisdictional claims in published maps and institutional affiliations.
Received: 16 February 2020 Accepted: 20 September 2020

Published online: 13 October 2020

\section{References}

1. Gruen RL, Brohi K, Schreiber M et al (2012) Haemorrhage control in severely injured patients. Lancet 380:1099-1108

2. Cole E, Weaver A, Gall L et al (2019) A decade of damage control resuscitation: new transfusion practice, new survivors, new directions. Ann Surg. https://doi.org/10.1097/SLA.0000000000003657

3. Brohi K, Gruen RL, Holcomb JB (2019) Why are bleeding trauma patients still dying? Intensive Care Med 45:709-711

4. CRASH-2 collaborators (2011) The importance of early treatment with tranexamic acid in bleeding trauma patients: an exploratory analysis of the CRASH-2 randomised controlled trial. Lancet 377:1096-1101

5. NICE recommendations Major Trauma (NG39) published February 2016. https://www.nice.org.uk/guidance/ng39. Accessed 1st of February 2020.

6. Holcomb JB, Tilley BC, Baraniuk S et al (2015) Transfusion of plasma, platelets, and red blood cells in a 1:1:1 vs a 1:1:2 ratio and mortality in patients with severe trauma. PROPPR Rand Clin Trial JAMA 313:471-482

7. Khan S, Davenport R, Raza I et al (2015) Damage control resuscitation using blood component therapy in standard doses has a limited effect on coagulopathy during trauma haemorrhage. Intensive Care Med 41:239-247

8. Balvers K, van Dieren S, Baksaas-Aasen K et al (2017) Combined effect of therapeutic strategies for bleeding injury on early survival, transfusion needs and correction of coagulopathy. Br J Surg 104:222-229

9. Davenport R, Manson J, De'Ath $\mathrm{H}$ et al (2011) Functional definition and characterization of acute traumatic coagulopathy. Crit Care Med 39:2652-2658

10. Johansson PI, Sørensen AM, Larsen CF et al (2013) Low haemorrhagerelated mortality in trauma patients in a Level I trauma centre employing transfusion packages and early thromboelastography-directed haemostatic resuscitation with plasma and platelets. Transfusion 53:3088-3099

11. Hagemo JS, Christiaans SC, Stanworth SJ et al (2015) Detection of acute traumatic coagulopathy and massive transfusion requirements by means of rotational thromboelastometry: an international prospective validation study. Crit Care 19:97

12. Holcomb JB, Minei KM, Scerbo ML et al (2012) Admission rapid thrombelastography can replace conventional coagulation tests in the emergency department. Ann Surg 256:476-486

13. Schochl H, Nienaber U, Hofer G et al (2010) Goal-directed coagulation management of major trauma patients using thromboelastometry (ROTEM)-guided administration of fibrinogen concentrate and prothrombin complex concentrate. Crit Care 14:R55

14. Tapia NM, Chang A, Norman M et al (2013) TEG-guided resuscitation is superior to standardized MTP resuscitation in massively transfused penetrating trauma patients. J Trauma Acute Care Surg 74:378-385

15. Einersen PM, Moore EE, Chapman MP et al (2017) Rapid-thrombelastography ( $r$-TEG) thresholds for goal-directed resuscitation of patients at risk for massive transfusion. J Trauma Acute Care Surg 82:114-119

16. Afshari A, Wikkelsoe A, Brok J, Moeller AM, Wetterslev J (2011) Thrombelastography (TEG) or thrombelastometry (ROTEM) to monitor haemotherapy versus usual care in patients with massive transfusion. Cochrane Database Syst Rev 16:CD007871. https://doi.org/10.1002/14651858. CD007871.pub2.

17. Da Luz LT, Nascimento B, Shankarakutty AK, Rizoli S, Adhikari NK (2014) Effect of thromboelastography $\left(\mathrm{TEG}^{\circledR}\right)$ and rotational thromboelastometry $\left(\right.$ ROTEM $\left.{ }^{\circledR}\right)$ on diagnosis of coagulopathy, transfusion guidance and mortality in trauma: descriptive systematic review. Crit Care 18:518

18. Hunt H, Stanworth S, Curry N, et al (2015) Thromboelastography (TEG) and rotational thromboelastometry (ROTEM) for trauma induced coagulopathy in adult trauma patients with bleeding (Review). Cochrane Database Syst Rev 16:CD 010438. https://doi.org/10.1002/14651858. CD010438.pub2.

19. Veigas PV, Callum J, Rizoli S, Nascimento B, da Luz LT (2016) A systematic review on the rotational thrombelastometry $\left(\right.$ ROTEM $\left.^{\circledR}\right)$ values for the diagnosis of coagulopathy, prediction and guidance of blood transfusion and prediction of mortality in trauma patients. Scand J Trauma, Resusc Emerg Med 24:114 
20. Winearls J, Reade M, Miles H et al (2016) Targeted coagulation management in severe trauma: the controversies and the evidence. Anesth Analg 123:910-924

21. Baksaas-Aasen K, van Dieren S, Balvers K et al (2019) Data-driven development of ROTEM and TEG algorithms for the management of trauma hemorrhage. Ann Surg 270:1178-1185

22. Baksaas-Aasen K, Gall L, Eaglestone S et al (2017) iTACTIC —implementing treatment algorithms for the correction of trauma-induced coagulopathy: study protocol for a multicentre, randomised controlled trial. Trials 18:486

23. Gennarelli TA, Champion HR, Sacco WJ, Copes WS, Alves WM (1989) Mortality of patients with head injury and extracranial injury treated in trauma centres. J Trauma 29:1193-1201

24. Frith D, Gosling JC, Gaarder C et al (2010) Definition and drivers of acute traumatic coagulopathy: clinical and experimental investigations. J Thromb Haemost 8:1919-1925

25. Gonzalez E, Moore EE, Moore HB et al (2016) Goal-directed hemostatic resuscitation of trauma-induced coagulopathy: a pragmatic randomized clinical trial comparing a viscoelastic assay to conventional coagulation assays. Ann Surg 263:1051-1059

26. Maegele M, Schöchl H, Menovsky T et al (2017) Coagulopathy and haemorrhagic progression in traumatic brain injury: advances in mechanisms, diagnosis, and management. Lancet Neurol 16:630-647
27. The CRASH-3 trial collaborators (2019) Effects of tranexamic acid on death, disability, vascular occlusive events and other morbidities in patients with acute traumatic brain injury (CRASH-3): a randomised, placebo-controlled trial. Lancet 394:1713-1723

28. Prehospital Tranexamic Acid Use for Traumatic Brain Injury (TXA): https:// clinicaltrials.gov/ct2/show/NCT01990768.Accessed 18st of August2020.

29. Steyerberg EW, Wiegers E, Sewalt C et al (2019) Case-mix, care pathways, and outcomes in patients with traumatic brain injury in CENTER-TBI: a European prospective, multicentre, longitudinal, cohort study. Lancet Neurol 18:923-934

30. Hawryluk GWJ, Aguilera S, Buki A et al (2019) A management algorithm for patients with intracranial pressure monitoring: the Seattle International Severe Traumatic Brain Injury Consensus Conference (SIBICC). Intensive Care Med 45:1783-1794

31. Chesnut R, Aguilera S, Buki A et al (2020) A management algorithm for adult patients with both brain oxygen and intracranial pressure monitoring: the Seattle International Severe Traumatic Brain Injury Consensus Conference (SIBICC). Intensive Care Med 46:919-929

32. Asehnoune K, Balogh Z, Citerio G et al (2017) The research agenda for trauma critical care. Intensive Care Med 43:1340-1351 